


\title{
Efecto de la gestión del factor humano en la flexibilidad y la efectividad organizacionales en PYMEs turísticas mexicanas
}

\author{
María Alondra de la Llave Hernández, Diana Donají del Callejo Canal \\ y Margarita Edith Canal Martínez
}

UNIVERSIDAD AUTÓNOMA DE CIUDAD JUÁREZ

PUBLICACIÓN AFILIADA A LA

RED IBEROAMERICANA DE ESTUDIOS DEL DESARROLLO 


\title{
UNIVERSIDAD AUTÓNOMA DE CIUDAD JUÁREZ
}

\author{
PUBLICACIÓN AFILIADA A LA \\ RED IBEROAMERICANA DE ESTUDIOS DEL DESARROLLO
}

\section{Universidad Autónoma de Ciudad Juárez} 2018-2024

Mtro. Juan Ignacio Camargo Nassar

Rector

Mtro. Daniel Alberto Constandse Cortez

Secretario General

Mtro. Santos Alonso Morales Muñoz Director del Instituto de Ciencias Sociales y Administración Mtro. Jesús Meza Vega

Director General de Comunicación Universitaria

Comité de Coordinación de la Red Iberoamericana de Estudios del Desarrollo 2018-2020

Dra. Paulina Sanhueza Martínez (Universidad de la Frontera, Chile) Coordinadora General

Dr. Ignacio Rodríguez Rodríguez (Universidad de la Frontera, Chile) Secretario general

Dra. Myrna Limas Hernández (Universidad Autónoma de Ciudad Juárez, México) Vocal de Organización Dr. Pablo Galaso Reca (Universidad de la República, Uruguay) Vocal de Organización

Dr. Luis Enrique Gutiérrez Casas Director y editor de Cuadernos de Trabajo Estudios Regionales en Economía, Población y Desarrollo

Comité editorial

Sección internacional Dra. Sofía Boza Martínez (Universidad de Chile, Chile) Dra. Olga Biosca Artiñano (Glasgow Caledonian University, Reino Unido)

Dra. Ángeles Sánchez Díez (Universidad Autónoma de Madrid, España) Dr. Thomas Fullerton Mankin (University of Texas at E1 Paso, Estados Unidos)

Dr. Adrián Rodríguez Miranda

(Universidad de la República, Uruguay) Dra. Ikuho Kochi

(Kanazawa University, Japón) Dr. Pablo Galaso Reca (Universidad de la República, Uruguay)

Sección local (Universidad Autónoma de Ciudad Juárez) Dra. Myrna Limas Hernández

Dra. Rosa María García Almada

Dr. Raúl Alberto Ponce Rodríguez

Dr. Isaac Leobardo Sánchez Juárez

Dr. Héctor Alonso Barajas Bustillos

Dr. Juan Carlos Medina Guirado Mtra. María Del Socorro Velázquez Vargas

Diseño de cubierta Abigail Bautista
Estudios Regionales en Economía, Población y Desarrollo. Cuadernos de Trabajo de la UACJ ISSN 2007-3739

Número 68. Marzo - Abril 2022

Efecto de la gestión del factor humano en la flexibilidad y la efectividad organizacionales en PYMEs turísticas mexicanas

María Alondra de la Llave Hernández, Diana Donají del Callejo Canal y Margarita Edith Canal Martínez

Universidad Autónoma de Ciudad Juárez

Estudios Regionales en Economía, Población y Desarrollo.

Cuadernos de Trabajo de la UACJ

Año 12, No. 68 marzo - abril 2022, es una publicación bimestral editada por la Universidad Autónoma de Ciudad Juárez a través del Cuerpo Académico de Estudios Regionales en Economía, Población y Desarrollo del Instituto de Ciencias Sociales y Administración. Redacción: Avenida Universidad y H. Colegio Militar, Zona Chamizal s/n., C.P. 32300, Ciudad Juárez, Chihuahua, México.

Teléfonos: (656) 688-38-00, ext.3792.Correo electrónico: igtz@uacj.mx. Editor responsable: Luis Enrique Gutiérrez Casas. Reserva de derechos al uso exclusivo: edición impresa, ISSN 2007-3739., edición digital, No. de reserva 04-2019-050218151500. Impresa por Studio Los Dorados, calle Del Campanario, número 820-2, Santa Cecilia, C.P. 32350, Cd. Juárez, Chihuahua. Distribuidor: Subdirección de Gestión de Proyecto y Marketing Editorial. Ave. Plutarco Elías Calles 1210, Foviste Chamizal, C.P. 32310 , Ciudad Juárez, Chihuahua. Este número se terminó de imprimir el 15 de febrero 2022 con un tiraje de 120 ejemplares.

Los ensayos publicados son responsabilidad exclusiva de sus autores.

Se autoriza la reproducción total o parcial bajo condición de citar la fuente.

\section{Registrada en: $\frac{\text { EBSCO }}{\text { Host }}$ RePEClex UAC] 侧 Revistas Electrónicas ๑ Dialnet}

Publicación afiliada a la Red Iberoamericana de Estudios del Desarrollo

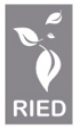

Universidad Autónoma de Ciudad Juárez

Ave Plutarco Elías Calles 1210

Foviste Chamizal, C.P. 32310

Ciudad Juárez, Chihuahua, México

www.uacj.mx

(C) Universidad Autónoma de Ciudad Juárez 
Estudios Regionales en Economía, Población y Desarrollo. Cuadernos de Trabajo, Universidad Autónoma de Ciudad Juárez, publicación afiliada a la Red Iberoamericana de Estudios del Desarrollo, número 68, marzo - abril de 2022, ISSN 2007-3739, pp. 3-22 México.

\title{
Efecto de la gestión del factor humano en la flexibilidad y la efectividad organizacionales en PYMEs turísticas mexicanas
}

\author{
María Alondra de la Llave Hernández *, Diana Donaji del Callejo Canal ** \\ y Margarita Edith Canal Martínez ***
}

\begin{abstract}
Resumen
La gestión del factor humano es un tema de creciente interés a nivel global y en prácticamente todos los sectores industriales. El propósito de este trabajo es identificar y medir el efecto de la Gestión de Factor Humano (GFH) en la Flexibilidad Organizacional (FL) y la Efectividad Organizacional (EF) a través de un estudio estadístico, derivado de una encuesta virtual, para la posterior definición de un modelo teórico de gestión del factor humano capaz de optimizar la flexibilidad y efectividad de las pequeñas y medianas empresas (PYMEs) hoteleras en México. Los resultados del estudio estadístico muestran que existe correlación entre la gestión del factor humano y la flexibilidad y efectividad organizacionales.
\end{abstract}

Palabras clave: Efectividad organizacional, flexibilidad organizacional, gestión, PYMEs.

\section{Effect of human factor management on organizational flexibility and effectiveness in Mexican tourism SMEs}

\begin{abstract}
The management of the human factor is a topic of growing interest globally and in practically all industrial sectors. The purpose of this work is to identify and measure the effect of Human Factor Management $(G F H)$ on Organizational Flexibility (FL) and Organizational Effectiveness (EF) through a statistical study, derived from a virtual survey, for the subsequent Definition of a theoretical model of human factor management capable of optimizing the flexibility and efficiency of small and medium-sized companies (SMEs) hotel in Mexico. The results of the statistical study show that there is a correlation between the management of the human factor and organizational flexibility and effectiveness.
\end{abstract}

Keywords: Organizational effectiveness, organizational flexibility, management, SMEs.

JEL: M12, M54, L25

Recibido en: noviembre de 2021. Aprobado en: enero de 2022.

\footnotetext{
* Candidata a doctorado por la Universidad Popular Autónoma del Estado de Puebla (México). Correo: mariaalondra.delallave@upaep.edu.mx. ORCID: 0000-0001-7206-1150.

** Profesora-Investigadora de la Universidad Veracruzana (México), adscrita al Instituto de Investigaciones y Estudios Superiores Económicos y Sociales (IIESES). Integrante del Laboratorio de Investigación en Estadística y Ciencia de Datos. Correo electrónico: ddelcallejo@uv.mx. ORCID: 0000-0003-4753-6577.

*** Profesora-Investigadora de la Universidad Veracruzana (México), adscrita al Instituto de Investigaciones y Estudios Superiores Económicos y Sociales (IIESES). Integrante del Laboratorio de Investigación en Estadística y Ciencia de Datos. Correo: mcanal@uv.mx. ORCID: 0000-0002-1258-5902.
} 


\section{$\rightarrow$ 1. Introducción.}

Las PYMEs son la principal fuente de empleo en el sector empresarial. En el área de la Organización para la Cooperación y Desarrollo Económico (OCDE), éstas representan casi la totalidad de la población empresarial, con un $60 \%$ del empleo total y generan en promedio entre el $50 \%$ y el $60 \%$ del valor agregado. Además, contribuyen con más de un tercio del Producto Interno Bruto (PIB) en las economías emergentes y en desarrollo, asimismo, representan el 34\% y el 52\% del empleo formal, respectivamente. Durante el período 2003-2016, en 132 países, el número total de empleados a tiempo completo en las PYMEs pasó de 79 millones a 156 millones (Cusmano, Koreen y Pissareva, 2018). En México, la estratificación bajo la cual se catalogan las Micro, Pequeñas y Medianas Empresas (MIPYMEs) se establece en la Ley para el Desarrollo de la Competitividad de la Micro, Pequeña y Mediana Empresa, de conformidad con los criterios indicados en la Tabla 1 (Secretaría de Economía, 2009).

\section{Tabla 1}

\section{Estratificación de MIPYMEs en México}

\begin{tabular}{|c|c|c|c|c|}
\hline Tamaño & Sector & $\begin{array}{l}\text { Número de } \\
\text { trabajadores }\end{array}$ & $\begin{array}{l}\text { Monto de ventas } \\
\text { anuales (mdp) }\end{array}$ & $\begin{array}{c}\text { Tope máximo } \\
\text { combinado } \\
\text { (Trabajadores) }(10 \%)+ \\
\text { (Ventas Anuales) }(90 \%)\end{array}$ \\
\hline Micro & Todas & $<=10$ & $<=\$ 4$ & 4.6 \\
\hline Pequeña & $\begin{array}{c}\text { Comercio } \\
\text { Industria y Servicios }\end{array}$ & $\begin{array}{l}11-30 \\
11-50\end{array}$ & $\$ 4.01-\$ 100$ & $\begin{array}{l}93 \\
95\end{array}$ \\
\hline Mediana & $\begin{array}{l}\text { Comercio } \\
\text { Servicios } \\
\text { Industria }\end{array}$ & $\begin{array}{l}31-100 \\
51-100 \\
51-250\end{array}$ & $\$ 100.01-\$ 250$ & $\begin{array}{l}235 \\
250\end{array}$ \\
\hline
\end{tabular}

Fuente: Elaboración propia con información de la Secretaría de Economía, 2009.

De acuerdo con el INEGI, en 2014 en México el 4.7\% de las unidades económicas eran pequeñas empresas y $0.8 \%$ medianas empresas (ver figura 1 ), mismas que emplean al $35.1 \%$ de la población económicamente activa (18.5\% en pequeñas empresas y $16.6 \%$ en medianas empresas (ver figura 2) y representan el $26.1 \%$ de la producción bruta total del país (INEGI, 2015). 


\section{Figura 1 \\ Porcentaje de establecimientos por número de personas ocupadas}

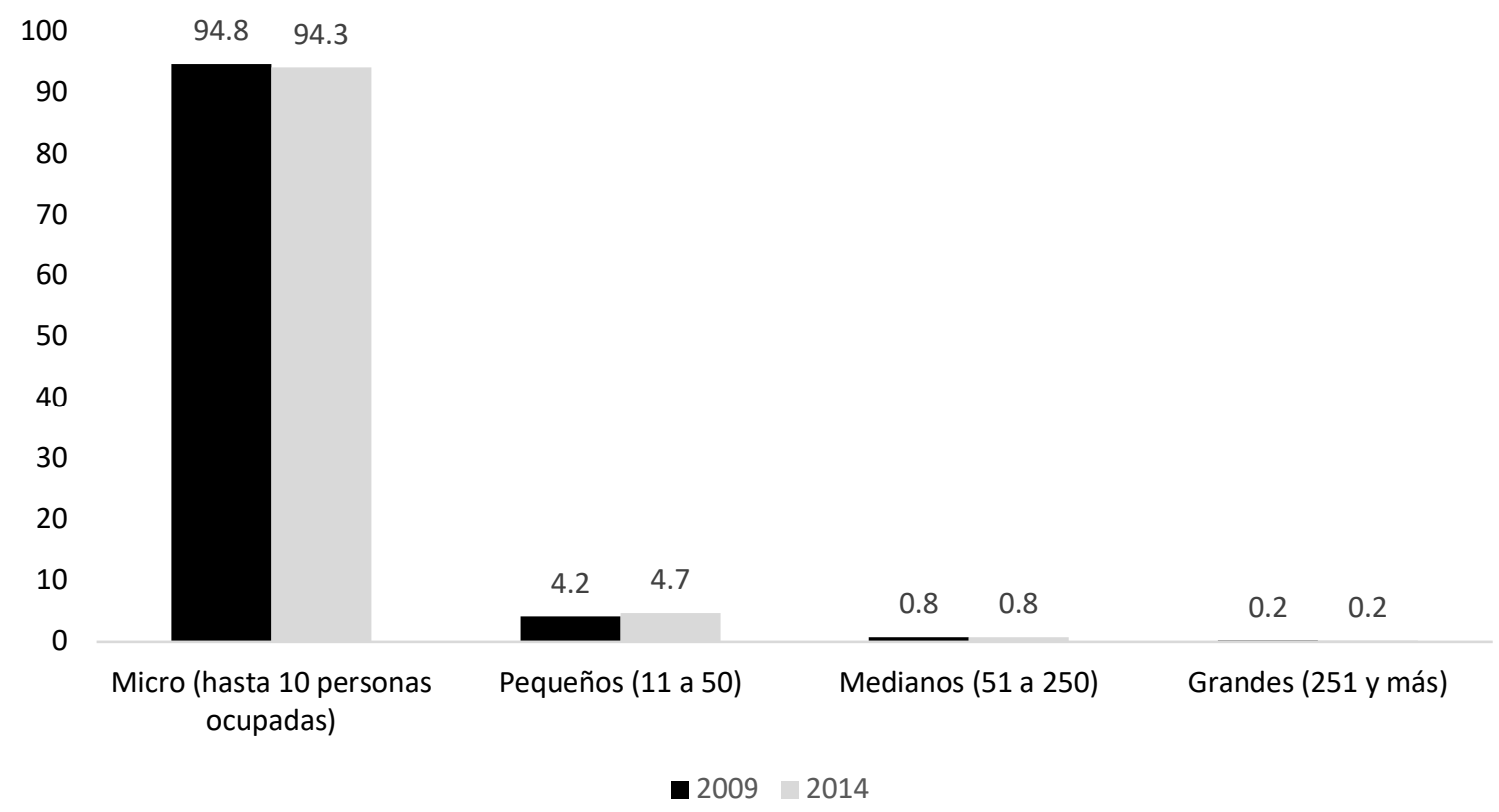

Fuente: Elaboración propia con datos de Instituto Nacional de Estadística y Geografía (INEGI), 2015.

Las PYMEs turísticas deben superar sus desafíos para desarrollar flexibilidad, eficacia y eficiencia (Nikolovski y Dimoska, 2016). Diversas técnicas de gestión, incluyendo gestión de calidad y Lean Manufacturing, están siendo utilizadas actualmente por diferentes y variados sectores para mejorar su productividad y flexibilidad (Sharma, Sachdeva y Gupta, 2017); sin embargo, se han encontrado hallazgos que evidencian dificultades en la implementación de este tipo de enfoques en PYMEs (Felizzola y Luna, 2014), siendo el factor humano el menos atendido, a pesar de que el respeto por la gente es el segundo pilar original de la filosofía Lean (Coetzee, van der Merwe y van Dyk, 2016).

Se identifica la necesidad de aplicar enfoques que agregan valor en las PYMEs de la industria turística; ya que estos pueden llevarla a atender mejor las necesidades de los clientes (Madgerova, Kyurova y Atanasova, 2016); además, es relevante para asegurar una implementación exitosa y no ignorar la dimensión valiosa de los trabajadores para lograr la calidad y satisfacer las necesidades del cliente (Snyder, Hedlund, Ingelsson y Bäckström, 2017). En vista de lo anterior, y con la intención de proveer a las PYMEs mexicanas del sector turístico en México dedicadas a la actividad de hotelería, de un modelo de gestión del factor humano, se busca inicialmente, la identificación y medición de la correlación entre la propia gestión del factor humano (GFH) con la flexibilidad (FL) y efectividad $(\mathrm{EF})$ organizacionales. 
Figura 2

\section{Porcentaje de personas ocupadas por estrato}

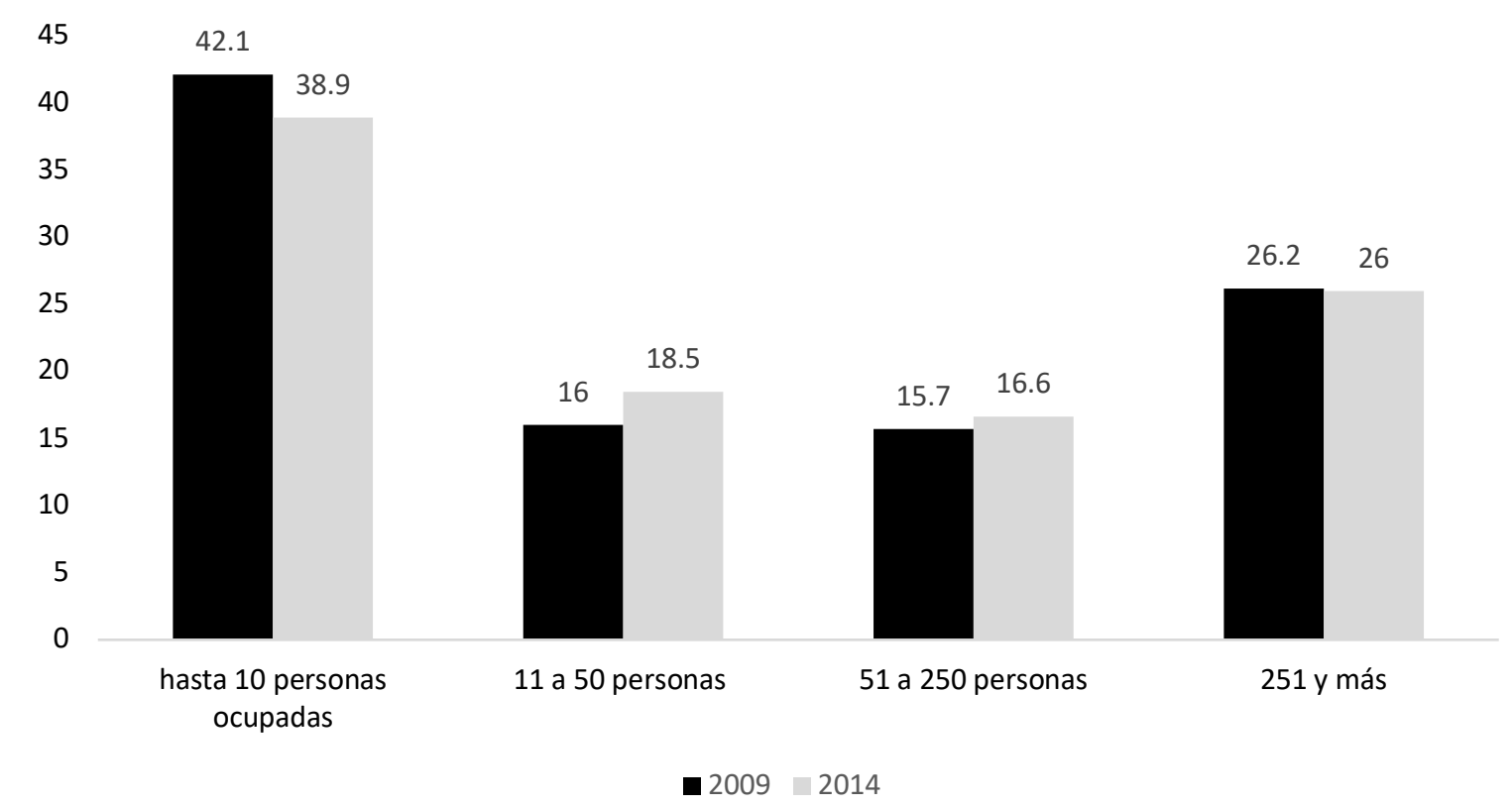

Fuente: Elaboración propia con datos de Instituto Nacional de Estadistica y Geografía (INEGI), 2015.

\section{La gestión del factor humano en la flexibilidad y efectividad organizacionales.}

\subsection{Modelos de gestión.}

Actualmente, las organizaciones tienen un reto mayor al que han tenido en el pasado para lograr los resultados que se esperan de ellas, deben ser rentables y competitivas en un entorno caótico y cambiante, donde los clientes son más exigentes y disponen de muchas opciones para cubrir sus necesidades. La competencia en estas condiciones requiere que las organizaciones, afronten los cambios que transforman aceleradamente a la sociedad, actualicen su tecnología para mejorar sus procesos y habiliten al personal, sin afectar negativamente el servicio; esto es posible, si la organización cuenta con un modelo de gestión sobre el cual soportar sus esfuerzos (CarballoMendívil, Arellano-González y Ríos-Vázquez, 2018).

La evolución de los modelos de gestión es el resultado procedente de la innovación y selección ascendente y descendente; se impulsa por las oportunidades de la revolución tecnológica y las restricciones de los paradigmas organizacionales establecidos. Cuando un modelo de gestión logra el 
dominio, se convierte en algo de sentido común y moldea la toma de decisiones; para que esto ocurra, es necesario un proceso de creación, teorización y difusión de las innovaciones organizacionales que configuren los modelos, conceptos y paradigmas organizacionales (Bodrozic and Adler, 2018).

Dos de las formas de gestión más utilizadas y reconocidas son el modelo PDCA (Plan - Do Check - Act), que es la piedra angular de los Sistemas de Gestión de los estándares ISO (Loján, Navarro \& Cagua 2017) y la Gestión Esbelta (Lean Management) que permite obtener valor, gestionando procesos altamente eficientes y persiguiendo la perfección (Cuatrecasas-Arbós, 2014). Debido a que la revolución tecnológica del automóvil y el petróleo fue seguida de la revolución tecnológica de la computación y las telecomunicaciones, y que no será la última, es necesario adecuar los paradigmas y modelos organizacionales a la realidad actual; esta adecuación a los modelos de gestión no puede basarse solamente en la selección de estrategias existentes, pues pronto se vería abrumada por los nuevos desarrollos en el mundo (Bodrozic and Adler, 2018; Brown \& Katz, 2019).

\subsection{Gestión del factor humano.}

Todas las organizaciones requieren de diversos recursos para efectuar sus tareas, siendo el recurso humano el factor clave, debido a su capacidad de aportar talento, esfuerzo y creatividad (Hernández y Quezada, 2016; Diaz-Fernández, Pasamar-Reyes y Valle-Cabrera, 2017). La gestión de las personas demanda una organización eficiente, así como diversas funciones que son completamente obligatorias para lograr los objetivos de la organización, cuando existe informalidad en las prácticas de recursos humanos se producen dificultades para enfrentar las crisis, mantener una orientación estratégica, y operar adecuadamente, principalmente en el caso de las PYMEs (Hernández y Quezada, 2016; Parnell, 2015).

Existen prácticas de probado impacto positivo en la gestión del factor humano, por ejemplo, se ha demostrado que las prácticas de flexibilidad organizacional, llevadas a la flexibilidad laboral impactan en el desempeño organizacional, al dotar a la organización de una fuerza laboral más productiva y competitiva (Madero y Barboza, 2015). Dado que el capital humano es un recurso clave para el éxito de la organización, su correcta gestión es muy significativa para la empresa, en la que el área de Recursos Humanos juega un papel importante (Diaz-Fernández et al., 2017).

Dos de las formas actuales y en auge, que adopta la gestión del factor humano son las denominadas Gestión Estratégica de Recursos Humanos (SHRM, Strategic Human Resource Management) y la Gestión del Talento (Gannon, Roper \& Doherty, 2015; Daubner-Siva, Ybema, Vinkenburg \& Beech, 2018).

La SHRM pretende lograr una ventaja competitiva para la organización a través de la gestión de los recursos humanos y las prácticas de recursos humanos, para lo que se vale de tres enfoques 
principales, que suelen usarse uno a la vez pero que no son mutuamente excluyentes. Los enfoques son, el universal o de mejores prácticas, el de mejor ajuste o contingencia y el enfoque basado en recursos (Gannon et al., 2015).

La Gestión del Talento (TM, Talent Management) se ocupa del impulso y la ejecución de una arquitectura de recursos humanos para ocupar puestos clave con empleados talentosos (de potencial y rendimiento altos), para así, mantener el avance competitivo de la organización. Actualmente la teoría de TM se concentra en tres discusiones principales: la primera relacionada con tener un enfoque exclusivo o inclusivo, en el sentido de poner el ojo sólo en empleados talentosos o en todos los integrantes de la organización; el segundo se enfoca en los resultados vislumbrados para los empleados bajo el supuesto del "efecto Pigmalión"; finalmente, el tercer tema acomete críticamente la combinación de prácticas y actividades de orientación unitaristas y gerencialistas, fundadas en el supuesto de ganancias mutuas, para el empleador y el empleado, pero con poca atención a la voz de los empleados (Daubner-Siva et al., 2018).

Sin embargo, los modelos actuales de gestión del factor humano, al igual que el resto de los sistemas sociales han perdido aptitud para su propósito al menos parcialmente, pues fueron diseñados para cumplir los requisitos de la "primera era de la máquina" y prácticamente se han mantenido sin cambios desde el siglo XIX y principios del siglo XX (Brown y Katz, 2019); como consecuencia, se ha presentado el hundimiento del diseño organizacional y de los procesos de gestión tradicionales, por lo que se debe reconocer la necesidad de nuevos conceptos administrativos adecuados a la disruptiva del siglo XXI (Mootte, 2014).

\subsection{Flexibilidad y efectividad organizacional.}

El término flexibilidad se refiere, entre otras cosas, a la disposición de cambio de forma de un objeto; a la adaptación "con facilidad a la opinión, a la voluntad o a la actitud de otro u otros" (RAE, 2019, p. s/n); y a la susceptibilidad "de cambios o variaciones según las circunstancias o necesidades" (RAE, 2019, p. s/n). De las acepciones mencionadas, es la tercera la que mejor se ajusta a la flexibilidad organizacional. Una cultura organizacional flexible se basa en el desarrollo del individuo y del grupo, en el aprendizaje y en la innovación, y esto es posible gracias al fomento de reglas ligeras, individualidad, participación, innovación, empoderamiento, trabajo en equipo, cambio y aprendizaje (Núñez, Mercado y Banegas, 2015).

La cuestión de la flexibilidad organizacional es particularmente importante en industrias dinámicas, donde el tipo de servicios apropiados hoy en día puede no parecerse mucho a los servicios que serán exigidos en el futuro próximo, dados los avances en el conocimiento y las tecnologías correspondientes. Por otro lado, la flexibilidad puede ser menos importante para otros tipos de 
proyectos, de naturaleza menos dinámica y poco cambiante, de hecho, es necesario que las organizaciones, no sean sólo flexibles, sino también efectivas, de una forma balanceada (Ross y Yan, 2015).

El termino efectividad se define como la "Capacidad de lograr el efecto que se desea o se espera" (RAE, 2019, p. s/n), partiendo de esta definición podemos decir que la efectividad organizacional es la capacidad de una organización para lograr los efectos o resultados esperados; no obstante, existen diversos modelos opuestos que han servido de base para estudiar la efectividad organizacional. Debido a que cada modelo utiliza su propia definición detallada de efectividad y también utiliza indicadores particulares para medir la efectividad organizacional, pudiendo ser estos cuantitativos o cualitativos (Martínez, Vera y Vera, 2014).

Gallo (2015), indica que la efectividad organizacional debe tener en cuenta el factor tiempo como la medida de indicación y recurso universal, pues es un factor de relevancia que incide en el desempeño de la organización, y concluye que se fundamenta en el grado de cumplimiento del resultado esperado, basado en la actividad de las personas que componen la organización y, en menor proporción, en los recursos tangibles.

En resumen, es posible definir la efectividad organizacional como la eficiencia y eficacia con que se alcanzan los objetivos organizacionales, mediante el diseño y funcionamiento actual de un sistema determinado, factores que, a su vez, se corresponden directamente con la manera en que interactúan los diferentes elementos del sistema organizativo. Por tanto, la efectividad organizacional consiste en identificar los fines de la organización y determinar si se han alcanzado adecuadamente (Camue, Carballal y Toscano, 2017).

\section{O 3. La PYME turística en México.}

Se entiende por turismo el fenómeno social, cultural y económico relacionado con el movimiento de las personas a lugares que se encuentran fuera de su lugar de residencia habitual, normalmente por motivos de ocio. De acuerdo con la Encuesta de Turismo de Internación, en septiembre de 2019, ingresaron a México más de siete millones de visitantes internacionales, dejando una derrama económica de más de mil cuatrocientos millones de dólares (INEGI, 2019); no obstante, es el turismo nacional el pilar del turismo en México, pues aporta 88 de cada 100 pesos consumidos por los turistas en el país, además de realizar una contribución económica en zonas del país no frecuentadas por el turismo internacional (OECD, 2017). 
En México, la política pública orientada a fortalecer el desarrollo de la actividad turística, promover la innovación en el sector, mejorar la calidad de los servicios turísticos y la competitividad del turismo nacional corren a cargo de la Secretaría de Turismo (SECTUR), mediante programas y acciones como el Fondo Nacional de Fomento al Turismo (FONATUR), Nombramiento de Pueblos Mágicos, Ángeles verdes, Representaciones Estatales de Turismo en México (visit México), Agendas Turísticas Náuticas, Distintivo ven a comer y Mercados Especializados (SECTUR, 2020).

De acuerdo con la Guía Oficial de Turismo en México VisitMéxico, los destinos turísticos en México se clasifican en Sol y Playa, Pueblos Mágicos, Grandes Ciudades, Ciudades Patrimonio y Destinos Culturales (VisitMéxico, 2020). Las ciudades patrimonio, cuentan con la declaración de la UNESCO como patrimonio mundial de la humanidad, México es el cuarto país en el mundo con más ciudades patrimonio, sólo después de España, Italia y Francia (ANCMPM, 2020).

Debido a la importancia económica de la actividad turística en México, la necesidad de proveer al sector con capital humano calificado, instituciones educativas de todo el país incluyen la formación en turismo dentro de su oferta educativa tanto en nivel técnico profesional asociado y licenciatura, como en posgrado (ver tabla 2).

En su Estudio de Política Turística en México, la OCDE, indica la importancia de la inversión y el financiamiento de las PYMEs en apoyo a la innovación de la oferta, pues constituyen un área prioritaria para fortalecer el sector turístico mexicano y aprovechar las oportunidades con fuerte potencial para el crecimiento económico. El estudio también indica que es imperativo, promover el espíritu empresarial y apoyar a las PyMES para que crezcan sus negocios y se integren a las cadenas de valor del turismo. Incentivar a las PyMES para integrarse en conglomerados que impulsen el desarrollo de destinos, mejorar su perfil hacia el mercado y crear capacidades de generar (y capturar) escala de mercado, eficiencia y capacidad organizativa para competir en las cadenas de suministro turístico (OECD, 2017).

Por otro lado, el estudio conducido por el Mtro. Jorge Trujillo Rincón (Trujillo-Rincón, 2015), dentro de los proyectos The Lab - Desarrollo de Sistemas de mercado para el Trabajo Decente de la Organización Internacional del Trabajo, muestra que entre los principales problemas que enfrentan las PYMEs turísticas, se encuentran la falta de capacitación y de motivación del personal, originada en deficiencias de gestión, y que redunda en alta rotación y fallas de calidad; además de identificar como un problema específico la "ausencia de gestión del recurso humano y precariedad en la calidad del trabajo" (p. 34) que ocasiona pobre productividad. 
Tabla 2

Programas educativos de nivel superior relacionados al turismo ofertados en México

\begin{tabular}{|l|l|}
\hline \multicolumn{1}{|c|}{ Sistema educativo } & \multicolumn{1}{c|}{ Programas educativos } \\
\hline $\begin{array}{l}\text { Universidades } \\
\text { Tecnológicas }\end{array}$ & $\begin{array}{l}\text { Licenciatura en Gestión y Desarrollo Turístico } \\
\text { Licenciatura en Turismo } \\
\text { TSU en Turismo área hotelería } \\
\text { TSU en Turismo área desarrollo de proyectos alternativos }\end{array}$ \\
\hline $\begin{array}{l}\text { Universidades } \\
\text { Politécnicas }\end{array}$ & Licenciatura en Administración de Empresas Turísticas \\
\hline $\begin{array}{l}\text { Tecnológico Nacional } \\
\text { Interculturales }\end{array}$ & $\begin{array}{l}\text { Licenciatura en Turismo } \\
\text { Licenciatura en Gastronomía }\end{array}$ \\
\hline $\begin{array}{l}\text { Autónomas estatales y } \\
\text { privadas }\end{array}$ & $\begin{array}{l}\text { Licenciatura en Turismo Alternativo } \\
\text { Licenciatura en Desarrollo Turístico Sustentable } \\
\text { Licenciatura en Administración Turística y Hotelera } \\
\text { Maestría en Ciencias para el Desarrollo, la Sustentabilidad y el Turismo } \\
\text { Maestría en gestión sustentable del turismo }\end{array}$ \\
\hline $\begin{array}{l}\text { Instituto Politécnico } \\
\text { Nacional }\end{array}$ & $\begin{array}{l}\text { Licenciatura en Turismo } \\
\text { Licenciatura en Turismo Sustentable } \\
\text { Maestría en Administración e Innovación del Turismo }\end{array}$ \\
\hline $\begin{array}{l}\text { Molegio de } \\
\text { Maestría en Paisaje y Turismo Rural }\end{array}$ \\
\hline
\end{tabular}

Fuente: Elaboración propia con información de: Colegio de Posgraduados (ColPos) (2020), Coordinación General de Educación Intercultural y Bilingüe (CGEIB) (2020), Dirección General de Universidades Tecnológicas y Politécnicas (CGUTyP) (2020), Instituto Politécnico Nacional (IPN) (2020), Tecnológico Nacional de México (TecNM) (2020), Universidad Autónoma de Nayarit (UAN) (2020), Universidad Autónoma de Querétaro (UAQ) (2020), Universidad de Guadalajara (UdG) (2020), Universidad de Morelia (UDEM) (2020), Universidad de Quintana Roo (UQRoo) (2020), Universidad del Valle de México (UVM) (2020).

Dentro del sector turístico, la hotelería es una industria relevante. Sobre la importancia económica de esta actividad y su participación en el Producto Interno Bruto, según la metodología de la Cuenta Satélite del Turismo, se destaca que México llegó a 7.7\% en ese indicador, el más alto de América Latina, y sólo por debajo de España, con 11\% en todos los países de Ibero-América (PérezGarcía, 2015).

El impacto de esta industria en los indicadores económicos depende del desempeño de las organizaciones. La efectividad organizacional y la calidad del servicio en la industria hotelera penden de varios factores, entre los que destaca en gran medida las interacciones positivas de los empleados con los clientes y de su disposición a participar en comportamientos de ciudadanía organizacional. Los roles de los empleados del hotel a menudo implican intensas interacciones interpersonales con los clientes y compañeros de trabajo. Las interacciones de los empleados de hotel con los clientes son esenciales para lograr con éxito sus objetivos organizacionales, por lo que la deseable y esencial 
inteligencia emocional para su línea de trabajo debe ser cultivada y administrada de manera efectiva. La gestión del factor humano en la industria hotelera debiera incluir, además de las pruebas de aptitud, la evaluación de la inteligencia emocional de los posibles empleados (Santos, Mustafa \& Cantu, 2018).

En cuanto a la generación de empleos, tenemos que el indicador promedio del empleo en la hotelería es de 0.61 personas por habitación. Por cada puesto de trabajo directo en este sector, se generan 2.5 indirectos, lo que refleja que sólo la hotelería ocuparía directa e indirectamente más de un millón 200 mil personas a nivel nacional. Además, existe un $20 \%$ adicional de empleos eventuales que se integran en las diferentes temporadas altas vacacionales, particularmente en destinos de playa (Pérez-García, 2015).

\section{O 4. Metodología.}

Se realizó un estudio estadístico para medir el efecto de la Gestión de Factor Humano (GFH) en la Flexibilidad Organizacional (FL) y la Efectividad Organizacional (EF), con la finalidad definir un modelo teórico de gestión del factor humano capaz de optimizar la flexibilidad y efectividad de las Pequeñas y Medianas Empresas (PYMEs) hoteleras en México y que pueda considerarse como un elemento clave para un buen desarrollo de dichas empresas.

\subsection{Datos.}

Se cuenta con una tabla de datos con 75 casos válidos y 44 variables, todas ellas de corte cualitativo. Las cuatro primeras variables son descriptivas de la persona que responde el cuestionario: Sexo (Hombre o Mujer); Nivel de estudios (Técnico, Licenciatura, Posgrado); y Edad ( $<30$ años, entre 30 y 45 años, $>45$ años). Se tienen 20 variables referidas a GFH; 10 variables referidas a FL y; 10 variables referidas a EF. Las 40 variables de GFH, FL, y EF fueron medidas en escala Likert, donde 5 significa muy de acuerdo y 1 nada de acuerdo.

\subsection{Cuestionario.}

El cuestionario fue publicado en la plataforma QuestionPro ${ }^{\circledR}$ y estuvo disponible al público durante 113 días naturales, comprendidos del 23 de marzo al 14 de julio de 2020.

Los ítems de cada uno de los bloques (GFH, FL, EF) se basaron en la revisión previa de 50 artículos sobre la gestión de recursos humanos, publicados entre 2015 y 2019 e indizados en al menos uno de los siguientes: DOAJ, ESCI, SJR, SSCI, SCIE y Scopus. 
El instrumento cuenta con validez:

i. De contenido. Para que el instrumento refleje dominio de qué tanto la medición representa a la variable (Hernández y Mendoza, 2018), el cuestionario incluye las prácticas de gestión del factor humano normalmente empleadas durante toda la estancia de éste en una organización, desde su incorporación y hasta su separación; así como a los comportamientos y conductas del personal relacionados con la flexibilidad y la efectividad organizacionales.

ii. De constructo. A partir de la revisión de literatura, se identificaron las prácticas comunes y recomendadas en la gestión del factor humano, así como su relación con las conductas reflejadas por el personal en cuanto a la flexibilidad y la efectividad organizacionales. Sobre esta base el constructo se estructura como sigue:

a. Bloque $1(\mathrm{GFH})$. Gestión del factor humano: prácticas en la gestión del recurso humano, desde la selección y hasta la separación del personal de la organización y en el orden Selección - Inducción - Capacitación - Evaluación - Reconocimiento Separación);

b. Bloque 2 (FL). Flexibilidad Organizacional: Adaptabilidad personal, puesto enriquecido, habilidades técnicas, habilidades sociales, rotación.

c. Bloque 3 (EF). Efectividad Organizacional: Enfoque al logro, trabajo en equipo, liderazgo, comunicación.

iii. Asociada con la opinión de expertos y de comprensión del instrumento. El instrumento fue revisado por expertos en gestión de factor humano y por expertos en diseño de instrumentos de evaluación y recolección de datos, que dieron sus observaciones en cuanto al contenido del cuestionario, la escala de evaluación de las afirmaciones, la cantidad, secuencia y codificación de ítems.

Adicionalmente, se utilizó como medida de congruencia interna el coeficiente Alpha de Cronbach, la prueba se realizó sobre una muestra de 30 cuestionarios respondidos por expertos en gestión del factor humano. El resultado para el cuestionario fue un Coeficiente de fiabilidad de 0.8909, que de acuerdo con Hernández y Mendoza (2018) es de confiabilidad elevada.

\subsection{Análisis estadístico.}

Se realizó un análisis descriptivo de las frecuencias de respuesta por cada categoría en cada una de las tres dimensiones: GFH, FL y EF. Posteriormente se realizó un análisis exploratorio de 
Análisis de correspondencias múltiples (MCA) con la finalidad de observar las relaciones al interior de las dimensiones. Se encontró que las variables utilizadas en GFH tenían una alta correlación positiva entre todas ellas; en la dimensión FL se localizó un punto atípico, con calificaciones muy por debajo del resto, se decidió analizar este caso de manera particular. En la dimensión EF, se encontraron relaciones positivas entre las 10 variables que lo conforman.

Este análisis sirvió de marco de referencia para la propuesta de calcular una sola variable por dimensión que fuera el resultado de la sumatoria de todas las respuestas obtenidas para esa dimensión. De manera que se obtuvieron tres nuevas variables con la siguiente fórmula:

$$
G F H T=\sum_{i=1}^{20} G F H_{i}, \quad F L T=\sum_{i=1}^{10} F L_{i}, \quad E F T=\sum_{i=1}^{10} F L_{i}
$$

\section{Dónde}

GFHT = el valor total de la gestión humana.Cuya escala es de 0 a 100.

$F L T=$ el valor total de la Flexibilidad Laboral. Cuya escala es de 0 a 50.

$E F T=$ el valor total de la efectividad laboral.Cuya escala es del 0 al 50.

$i=$ es el número total de preguntas por cada dimensión.

Todos los análisis estadísticos fueron realizados con R studio, versión 1.2.5001 (RStudio Team, 2020).

\subsection{Modelo lineal.}

Se calcularon las medidas de tendencia central y las medidas de dispersión de cada una de las tres nuevas variables: GFHT, FLT, EFT y se utilizaron gráficas de cajas e histogramas para ver la distribución de cada una de las variables. Posteriormente se calculó la matriz de correlación de Pearson para conocer las correlaciones entra las variables y ulteriormente se propusieron dos modelos de regresión lineal simple para conocer la relación entre: 1) GFHR (como variable independiente) y FLT (como variable dependiente) y 2) GFHT (como variable independiente) y EFT (como variable dependiente).

De manera que los modelos propuestos fueron los siguientes:

$$
\begin{array}{ll}
\text { Modelo 1. } & \widehat{F L T}=\widehat{\beta_{0}}+\widehat{\beta_{1}} G F H T \\
\text { Modelo 2. } & \widehat{E F T}=\widehat{\beta_{0}}+\widehat{\beta_{1}} G F H T
\end{array}
$$


El supuesto de normalidad fue probado con la prueba de Shapiro-Wilks y en ambos modelos se cumplió $\left(p-\right.$ valor $_{\text {modelo } 1}<0.8457, p-$ valor $\left._{\text {modelo } 2}<0.9556\right)$. La homogeneidad de varianza fue verificada mediante la prueba de Breusch-Pagan, para ambos modelos se cumple el supuesto $\left(p-\right.$ valor $_{\text {modelo } 1}<0.3848, p-$ valor $\left._{\text {modelo } 2}<0.06\right)$.

\section{$\supset$ 5. Resultados.}

\subsection{Estadística descriptiva.}

Como se observa en la Tabla 3, los valores de GFHT alcanzan un mínimo de 44 puntos y un máximo de 100 puntos, con una media y una mediana muy centradas alrededor de 80 puntos. FLT y EFT tienen un mínimo, un máximo y una media muy parecida, pero una desviación estándar que es menor en FLT que en EFT.

Tabla 3

Estadísticas descriptivas por variable

\begin{tabular}{lrrrrr}
\hline Dimensión & \multicolumn{1}{c}{ Mín. } & Mediana & Media & Máx. & Est. Desv. \\
\hline GFHT & 44 & 80 & 77.8 & 100 & 12.9 \\
FLT & 30 & 41 & 40.9 & 49 & 3.77 \\
EFT & 28 & 42 & 40.1 & 50 & 5.29 \\
\hline
\end{tabular}

Fuente: Elaboración propia, en base al análisis estadístico.

\subsection{Análisis de correlación.}

El resultado de la matriz de correlaciones de Pearson muestra que existe una relación positiva entre GFHT y FLT ( $\rho=0.36$ ), aunque la correlación no es alta, si es suficiente para proponer un modelo de regresión lineal. El coeficiente de correlación de Pearson entre GFHT y EFT muestra una alta relación positiva entre estas dos variables $(\rho=0.58)$. Estos primeros resultados apoyan la idea de que a medida que crece el GHFT también crece el FLT y EFT.

a. Influencia de GFHT en FLT.

Los resultados del primer modelo (Tabla 4), muestran que el 12\% de la variación de EFT es explicada por GFHT, este resultado es lógico ya que además de GFHT hay otra serie de variables que intervienen en EFT. La relación es significativa en un 95\% de confianza y el valor del coeficiente estimado tanto del intercepto como del GFHT es significativo. Esto indica que por cada incremento 
en un punto de GFHT, FLT aumentará en promedio en 0.11 puntos. En la Figura 3 se puede apreciar la recta de regresión entre estas dos variables.

Tabla 4

\section{Resultados del modelo 1}

\begin{tabular}{lrr}
\hline \multicolumn{3}{c}{ Variable dependiente: FLT } \\
\multicolumn{1}{c}{ Modelo 1 } & Coeficiente & Error est. \\
\hline Intercepto & $32.61 * * *$ & 2.52 \\
GFHT & $0.11 * *$ \\
$\mathrm{R}^{2}$ ajustada & 0.1213 \\
$\mathrm{~F}$ & $11.08^{* *}$ \\
\hline$* * *$ p-valor $<0.001, * *$ p-valor $<0.01, * \mathrm{p}-\mathrm{valor}<0.05$. \\
Fuente: Elaboración propia, en base al análisis de correlación.
\end{tabular}

Figura 3

\section{Gráfico de dispersión y recta de regresión entre GFHT y FLT}

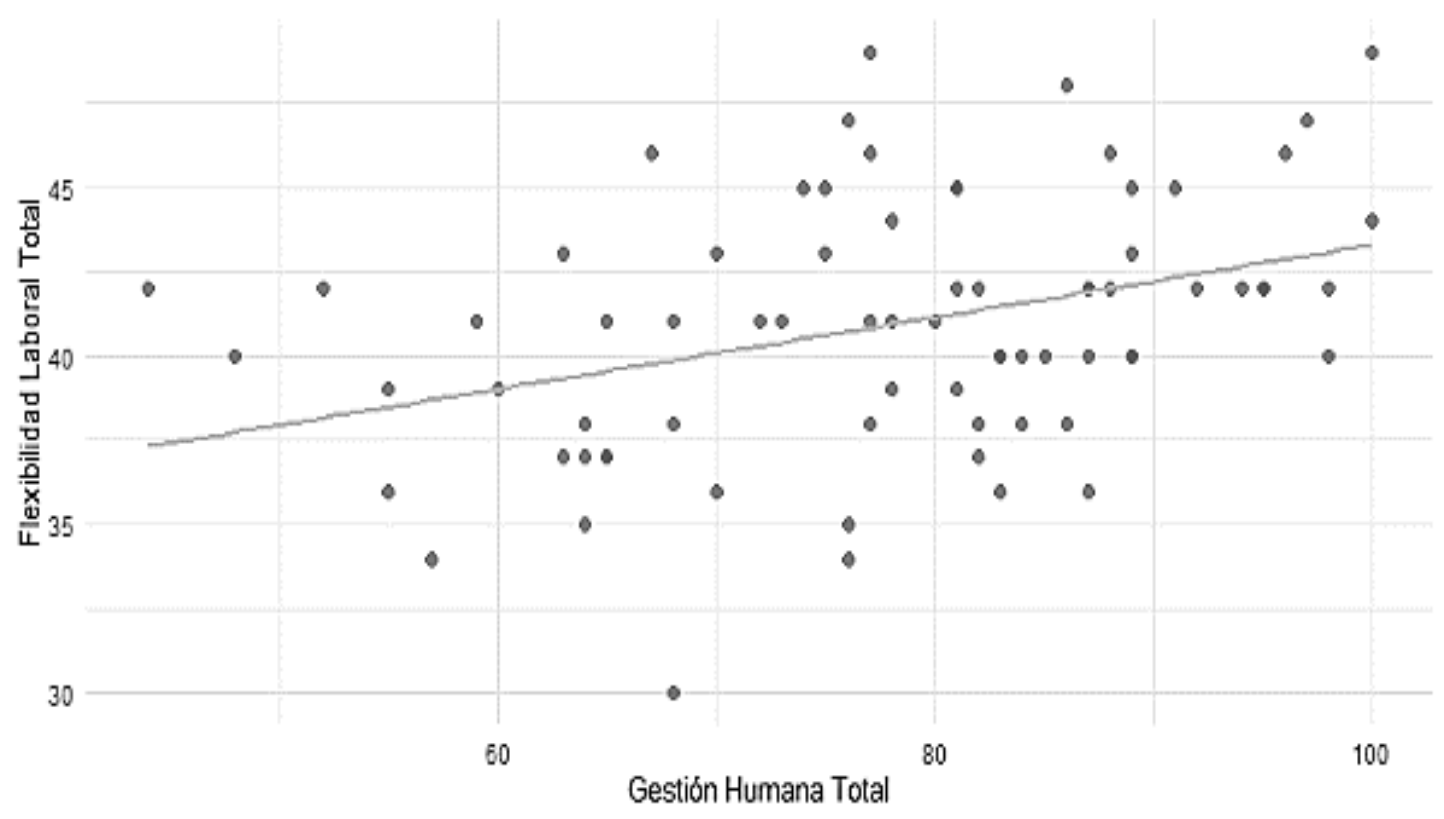

Fuente: Elaboración propia, en base al análisis de correlación. 
b. Influencia de GFHT en EFT.

Los resultados del modelo 2 se muestran en la Tabla 5 y en la Figura 4 . En este caso el $33 \%$ de la variación de EFT es explicada por GFHT. La relación es significativa en un 95\% de confianza y el valor del coeficiente estimado tanto del intercepto como del GFHT es significativo. Esto indica que por cada incremento en un punto de GFHT, EFT aumentará en promedio en 0.24 puntos. En la figura 4 , se puede apreciar la recta de regresión entre estas dos variables.

Tabla 5

\section{Resultados del modelo 2}

\begin{tabular}{lrr}
\hline \multicolumn{3}{c}{ Variable dependiente: FLT } \\
\multicolumn{1}{c}{ Modelo 1 } & Coeficiente & Error est. \\
\hline Intercepto & $22.07^{* * *}$ & 3.09 \\
GFHT & $0.24^{* * *}$ & 0.04 \\
$\mathrm{R}^{2}$ ajustada & 0.3314 & \\
$\mathrm{~F}$ & $37.18^{* *}$ \\
\hline ***p-valor $<0.001, * * \mathrm{p}-\mathrm{valor}<0.01$, *p-valor $<0.05$. \\
Fuente: Elaboración propia, en base al análisis de correlación.
\end{tabular}

Figura 4

\section{Gráfico de dispersión y recta de regresión entre GFHT y EFT}

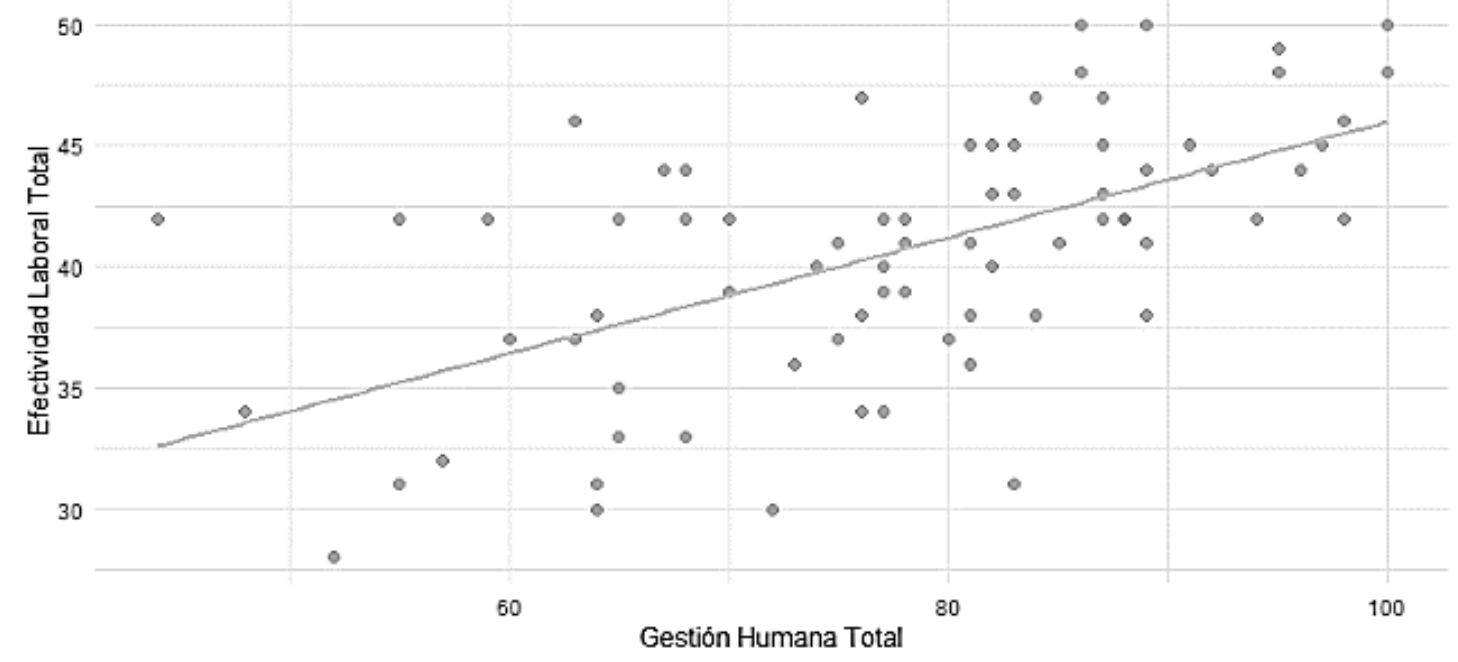

Fuente: Elaboración propia, en base al análisis de correlación. 


\section{○. Conclusiones.}

Hay que considerar que los resultados aquí presentados provienen de una encuesta, por lo que este estudio es de carácter exploratorio. Sin embargo, se evidencia que la Gestión de Factor Humano (GFH) tiene un efecto tanto en la Flexibilidad Organizacional (FL) como en la Efectividad Organizacional (EF). La relación lineal es más fuerte para la efectividad organizacional que para la flexibilidad.

Entre los estudios consultados para esta investigación, en lo que al sector industrial estudiado como contexto específico se refiere, se tienen aplicaciones en servicios bibliotecarios (Hernández y Quezada, 2016; Yeh y Walter, 2016), servicios de salud (Kash, Spaulding, Johnson y Gamy, 2014), logística (Oláh, Karmazin, FarKasné y Popp, 2017) y manufactura en el sector de la aeronáutica (Martínez-Jurado, Moyano-Fuentes y Jerez-Gómez, 2013). Al enfocarse esta investigación en el sector turístico, es posible comparar y contrastar la información disponible sobre la gestión del factor humano entre diferentes sectores industriales, posibilitando a futuro, la identificación de mejores prácticas de un sector que puedan ser adoptadas por otro.

Las investigaciones realizadas hasta el momento son en su mayoría exploratorias, por lo que dejan áreas de oportunidad y vacíos en la ciencia aún pendientes por ser atendidos. Martínez-Jurado et al. (2013), proponen que se realicen más investigaciones para tratar de medir la intensidad de las relaciones que se han identificado entre los factores explicativos y sus factores principales, durante el proceso de adopción y el proceso de implementación, para el cambio cultural. Por otro lado, Kash et al. (2014), plantean preciso dar atención formal a la interdependencia esencial de las iniciativas de cambio múltiple como factores de éxito mutuos, ya que puede resultar valioso a medida que las organizaciones persiguen un cambio estratégico.

También se identifica como necesario, realizar estudios adicionales que utilicen enfoques correlacionales y explicativos que apoyen el desarrollo de proyectos para mejorar las condiciones del personal y ayudar a llenar el vacío teórico y práctica en este campo. Los autores plantean que la obtención de estos datos servirá para establecer un modelo de desarrollo de recursos humanos que pueda replicarse en el futuro (Hernández y Quezada, 2016).

Como propuestas a futuro, se puede plantear indagar que otras variables están apoyando a la FL e incluirlas en el modelo; así como, aplicar el instrumento empleado en este estudio en una muestra del sector turístico más amplia para identificar particularidades entre diferentes actividades productivas del mismo sector (por ejemplo: hotelería, transporte y eventos recreativos, entre otros) y que requieren atención específica para lograr la flexibilidad y efectividad organizaciones; e identificar diferencias en la GFH entre sectores industriales. La identificación de necesidades y prácticas 
específicas por sector y actividad puede conducir a la generación de un modelo de gestión del factor humano, diseñado como traje a la medida de las empresas de un sector particular, en el caso de esta investigación, para las pequeñas y medianas empresas turísticas mexicanas dedicadas a la hotelería. 


\section{Bibliografía y referencias documentales}

Asociación Nacional de Ciudades Mexicanas del Patrimonio Mundial, AC. (ANCMPM) (2020). https://ciudadespatrimonio.mx/ancmpm-info/.

Bodrozic, Z. and Adler, P.S. (2018). The Evolution of Management Models: A Neo-Schumpeterian Theory. Administrative Science Quarterly, Vol. 63(1), 85-129. DOI: 10.1177/0001839217704811.

Brown, T. y Katz, B. (2019). Change by Design, Revised and Updated. HarperCollins Publishers. Nueva York, USA.

Camue Álvarez, A., Carballal del Río, E. y Toscano Ruiz, D.F. (2017). Concepciones teóricas sobre la efectividad organizacional y su evaluación en las universidades. Cofin Habana, Volumen 11, Número 2, 136-152. http://scielo.sld.cu/scielo.php?script=sci_arttext\&pid=S2073-60612017000200010

Carballo-Mendívil, B., Arellano-González, A. y Ríos-Vázquez, N. J. (2018). La gestión de procesos esbeltos como principio de mejora. Un caso aplicado a una comercializadora. 3C Empresa: Investigación y pensamiento crítico. Volumen $7, \quad$ número $3, \quad 60-81$. DOI: http://dx.doi.org/10.177993/3cemp.2018.070335.60-81/

Coetzee, R; van der Merwe, K. y van Dyk, L. (2016). Lean implementation strategies: how are the toyota way principles addressed? South African Journal of Industrial Engineering, Vol 27(3), 79-91.

https://www.researchgate.net/publication/311433103_Lean_implementation_strategies_How_are_the_ Toyota_Way_principles_addressed

Colegio de Postgraduados, Campus Córdoba (ColPos) (2020). https://www.colpos.mx/posgrado/ptr/

Coordinación General de Educación Intercultural y Bilingüe (CGEIB) (2020). https://eib.sep.gob.mx/diversidad/

Cuatrecasas-Arbós, Ll. (2014). Lean Management: la excelencia empresarial basada en obtener grandes resultados con pocos recursos. Revista de Contabilidad y Dirección, Vol. 19, pp. 51-70.

Cusmano, L., M. Koreen and L. Pissareva (2018), 2018 OECD Ministerial Conference on SMEs: Key Issues Paper, OECD SME and Entrepreneurship Papers, No. 7, OECD Publishing, Paris. http://dx.doi.org/10.1787/90c8823c-en

Daubner-Siva, D., Ybema, S., Vinkenburg, C.J. \& Beech, N. (2018). The talent paradox: talent management as a mixed blessing. Journal of Organizational Ethnography, Vol. 7 Issue: 1, pp.74-86, https://doi.org/10.1108/JOE-01-2017-0002

Diaz-Fernandez, M., Pasamar-Reyes, S. y Valle-Cabrera, R. (2017). Human capital and human resource management to achieve ambidextrous learning: A structural perspective. BRQ Business Research Quarterly, Volume 20, Issue 1, 63-77. https://doi.org/10.1016/j.brq.2016.03.002

Dirección General de Universidades Tecnológicas y Politécnicas (DGUTyP) (2020), https://dgutyp.sep.gob.mx/

Felizzola Jiménez, H. y Luna Amaya, C. (2014). Lean Six Sigma en pequeñas y medianas empresas: un enfoque metodológico. Ingeniare. Revista Chilena de Ingeniería, 22(2), 263-277. https://doi.org/10.4067/S071833052014000200012

Gallo Ramírez, C.M. (2015). Gestión empresarial: el liderazgo y la efectividad organizacional. Innovación Empresarial, Vol. 1, $\mathrm{N}^{\circ} 1,35-42$.

Gannon, J.M., Roper, A. \& Doherty, L. (2015). Strategic human resource management: Insights from the international hotel industry. International Journal of Hospitality Management, 47, 65-75. http://dx.doi.org/10.1016/j.ijhm.2015.03.003

Hernández Pacheco, F. y Quezada Escamilla, D. (2016). Human resources in Mexican public libraries: and exploratory inquiry. Investigación Bibliotecológica, $30 \quad$ (68), 17-50. https://doi.org/10.1016/j.ibbai.2016.06.002

Hernández Sampieri, R. y Mendoza Torres, C.P. (2018). Metodología de la investigación, las rutas cuantitativa, cualitativa y mixta. McGraw Hill Education. México.

Instituto Nacional de Estadística y Geografía (INEGI) (2019). INEGI Encuestas de viajeros internacionales (EVI). https://www.inegi.org.mx/temas/turismo/

Instituto Nacional de Estadística y Geografía (INEGI) (2015). Resultados definitivos. Censos económicos 2014. https://www.inegi.org.mx/app/saladeprensa/noticia.html?id= 1917

Instituto Politécnico Nacional (IPN) (2020). https://www.ipn.mx/oferta-educativa/educacion-superior/vercarrera.html? $\mathrm{lg}=$ es\&id $=60$ 
Kash, B. A; Spaulding, A; Johnson, C.F; y Gamy, L. (2014). Success Factors for Strategic Change Initiatives: A Qualitative Study of Healthcare Administrators' Perspectives. Journal of Healthcare Management 59 (1) 65-81. DOI: 10.1097/00115514-201401000-00011

Loján Granda, E., Navarro Espinosa, J. \& Cagua Vásquez, C. (2017). Modelo de evaluación de gestión de continuidad del negocio basado en la norma ISO 22301:2012. Revista ESPACIOS, Vol. 38 (No. 54), 116. https://www.revistaespacios.com/a17v38n54/a17v38n54p03.pdf

Madero Gómez, S.M. y Barboza, G.A. (2015). Interrelación de la cultura, flexibilidad laboral, alineación estratégica, innovación y rendimiento. Contaduría y Administración, 60, 735-756. http://dx.doi.org/10.1016/j.cya.2014.08.001

Madgerova, R. H.; Kyurova, V.V; Atanasova, A. V. (2016). Application of the Lean concept as a prerrequisite for a tourist business development. Economic Processes Management, 4, 1-19. https://essuir.sumdu.edu.ua/bitstreamdownload/123456789/49862/1/Madherova_turystychnyi_biznes.pdf

Martínez-Jurado, P. J., Moyano-Fuentes, J. y Jerez-Gómez, P. (2013). Human resource management in Lean Production adoption and implementation processes: Success factors in the aeronautics industry. $B R Q$ Business Research Quarterly, 17, 47-68. http://dx.doi.Org/10.1016/j.cede.2013.06.004

Martínez Méndez, R., Vera Muñoz, M.A.M. y Serafín Vera Muñoz, J.G. (2014). Cultura organizacional y efectividad en las pequeñas empresas constructoras de Puebla, México. Revista Internacional Administracion y Finanzas, Volumen 6 (3), 71-83. http://www.theibfr2.com/RePEc/ibf/riafin/riaf-v6n32013/RIAF-V6N3-2013-6.pdf

Mootte, I. (2014). Design Thinking para la Innovación Estratégica. 2da Edición, Empresa Activa. Valladolid, España.

Nikolovski, B., y Dimoska, T. (2016). Entrepenuarial design of quality initiatives in small tourism business, 185-196. https://doi.org/10.20544/HORIZONS.A.19.1.16.P17

Núñez Ramírez, M.A., Mercado Salgado, P., Banegas Rivero, R.A. (2015). Relación entre Cultura Organizacional (flexible y rígida) y Capital Intelectual. Conciencia Tecnológica, 49, 4-11.

Organización para la Cooperación y el Desarrollo Económicos (OECD) (2017). Tourism Policy Review of Mexico, OECD Studies on Tourism. OECD Publishing, Paris. DOI: http//dx.doi.org/10.1787/9789264266575-en

Oláh, J., Karmazin, G., FarKasné FeKete, M. y Popp, J. (2017). An examination of trust as a strategical factor of success in logistical firms. Business: Theory and practice, 18, 171-177. https://doi.org/10.3846/btp.2017.018

Parnell, J.A. (2015). Crisis Management and Strategic Orientation in Small and Medium-Sized Enterprises (SMEs) in Peru, Mexico, and the United States. Journal of Contingencies and Crisis Management. Volume 23 Number 4, 221-233. http://doi.org/10.1111/1468-5973.12060

Pérez-García, H. (2015). Historia de hotelería y turismo en México. Universidad de Guadalajara. Puerto Vallarta, Jalisco.

Real Academia Española (2019). Diccionario de la Lengua Española, Edición del Tricentenario. https://dle.rae.es/

Ross, T.W. y Yan, J. (2015). Comparing Public-Private Partnerships and Traditional Public Procurement: Efficiency vs. Flexibility, Journal of Comparative Policy Analysis: Research and Practice, DOI: $10.1080 / 13876988.2015 .1029333$

RStudio Team (2020). RStudio: Integrated Development for $R$. RStudio, PBC. Boston, MA URL http://www.rstudio.com/.

Santos, A., Mustafa, M., \& Cantu, A. A. (2018). Exploring the direct and indirect effects of emotional intelligence and frequency of customer contact on organisational citizenship behaviours among hotel employees in Mexico. International Journal of Work Organisation and Emotion, 9(3), 243-263. doi:10.1504/IJWOE.2018.094681

Secretaría de Economía. (2009). Acuerdo por el que se establece la estratificación de las micro, pequeñas y medianas empresas. Diario Oficial de la Federación. http://dof.gob.mx/nota_detalle.php?codigo $=5096849 \&$ fecha $=30 / 06 / 2009$

Secretaria de Turismo (SECTŪR) (2020). ¿Qué hacemos? https://www.gob.mx/sectur/que-hacemos

Sharma, R., Sachdeva, A., y Gupta, A. (2017). Commonality Amongst Various Lean Manufacturing Techniques: An Investigation in the Indian Automobile Industry. The IUP Journal of Operations Management, 16(2), $21-35$. 
Snyder, Kristen; Hedlund, Christer; Ingelsson, Pernilla y Bäckström, Ingela (2017). Storytelling: a cocreative process to support value-based leadership. International Journal of Quality and Service Sciences, 9 (3/4), 484-497, https://doi.org/10.1108/IJQSS-02-2017-0009

Tecnológico Nacional de México (TecNM) (2020). https://www.tecnm.mx/?vista=Licencia-turas

Trujillo-Rincón, Jorge (2015). PYMES más competitivas para la creación de más y mejores empleos, análisis de la cadena de valor del sector turístico de San Cristóbal de las Casas, Chiapas, México. THE LAB Desarrollo de sistemas de mercado para el trabajo decente. Organización Internacional del Trabajo. https://www.academia.edu/30338912/PYMES_M\%C3\%81S_COMPETITIVAS_PARA_LA_CREACI $\%$ C3\%93N_DE_M\%C3\%81S_Y_MEJORES_EMPLEOS_An\%C3\%A1lisis_de_la_cadena_de_valor_ del_sector_tur\%C3\%ADstico_de_San_Crist $\%$ C3\%B3bal_de_las_Casas_Chiapas_M\%C3\%A9xico

Universidad Autónoma de Nayarit (UAN) (2020). https://www.uan.edu.mx/es/unidad-academica-de-turismo

Universidad Autónoma de Querétaro (UAQ) (2020). https://www.uaq.mx/index.php/carreras/licenciaturas/fcya-2/licenciatura-en-gestion-del-turismocultural-y-natural

Universidad de Guadalajara (UdG) (2020). http://www.cucea.udg.mx/es/ofertaacademica/licenciaturas/turismo

Universidad de Morelia (UDEM) (2020). https://www.udemorelia.edu.mx/ofertaacademica/licenciatura/turismo-cultural/

Universidad de Quintana Roo (UQRoo) (2020), https://www.uqroo.mx/planes-deestudio/licenciaturas/chetumal/licenciatura-en-gestion-del-turismo-alternativo/

Universidad del Valle de México (UVM) (2020). https:/uvm.mx/oferta-academica/licenciaturasingenierias/hospitalidad-turismo-gastronomia/licenciatura-en-administracion-turistica-y-hotelera

Visit México (2020). Conoce México y sus 32 Estados. https://www.visitmexico.com/es/donde-ir/

Yeh, S. T y Walter, Z. (2016). Critical Success Factors for Integrated Library System Implementation in Academic Libraries: A Qualitative Study. Information Technology and Libraries, 27-42. doi:10.6017/ital.v35i2.9255. 


\section{Números anteriores}

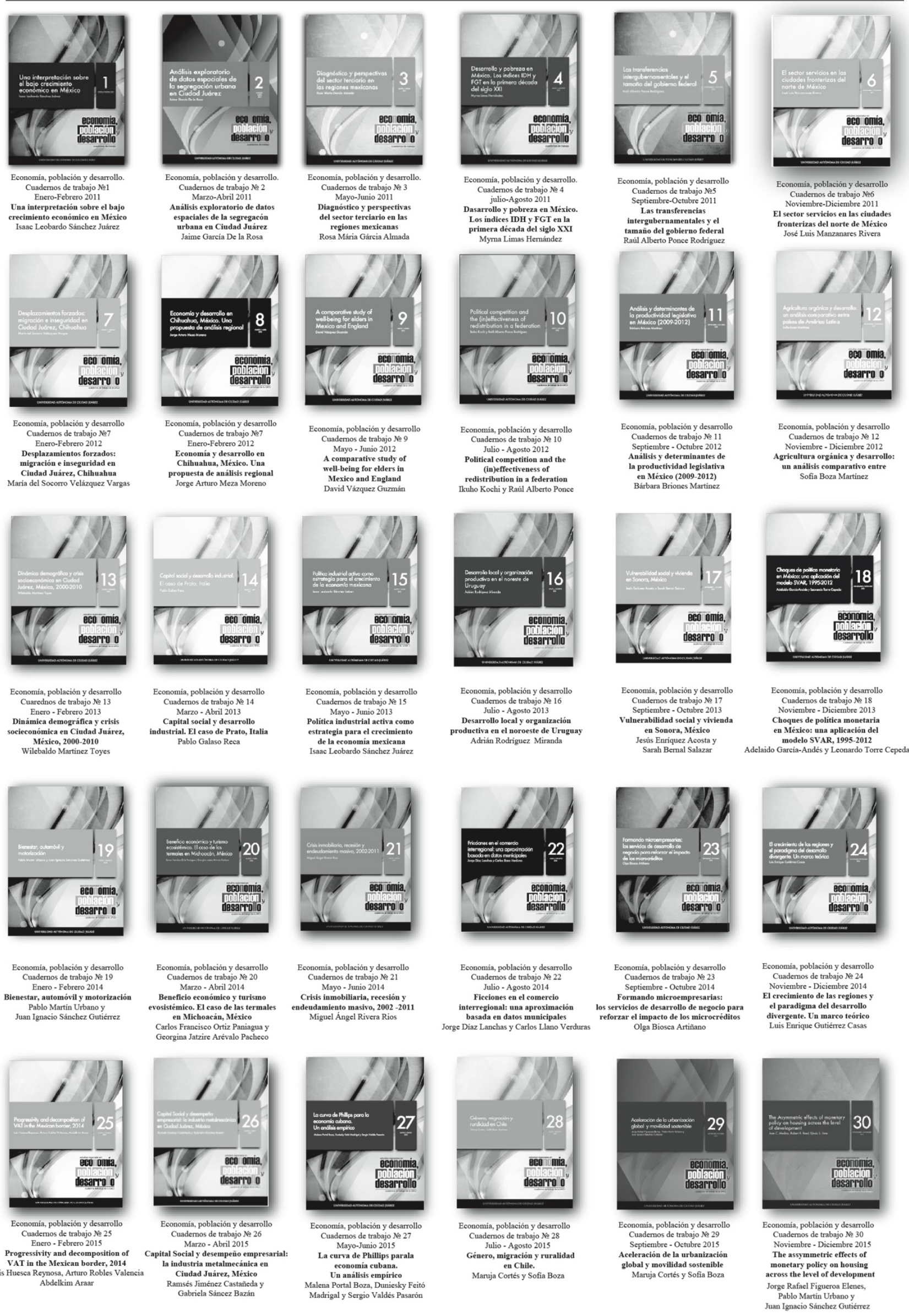



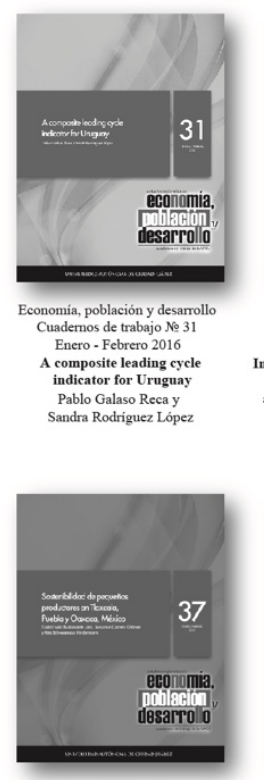

Economia, población y desarrollo

Cuademos de trabajo № 37 Sostenibilidad de pequeños

productores en Tlaxcala,

Benjamin Carrera Chávez y
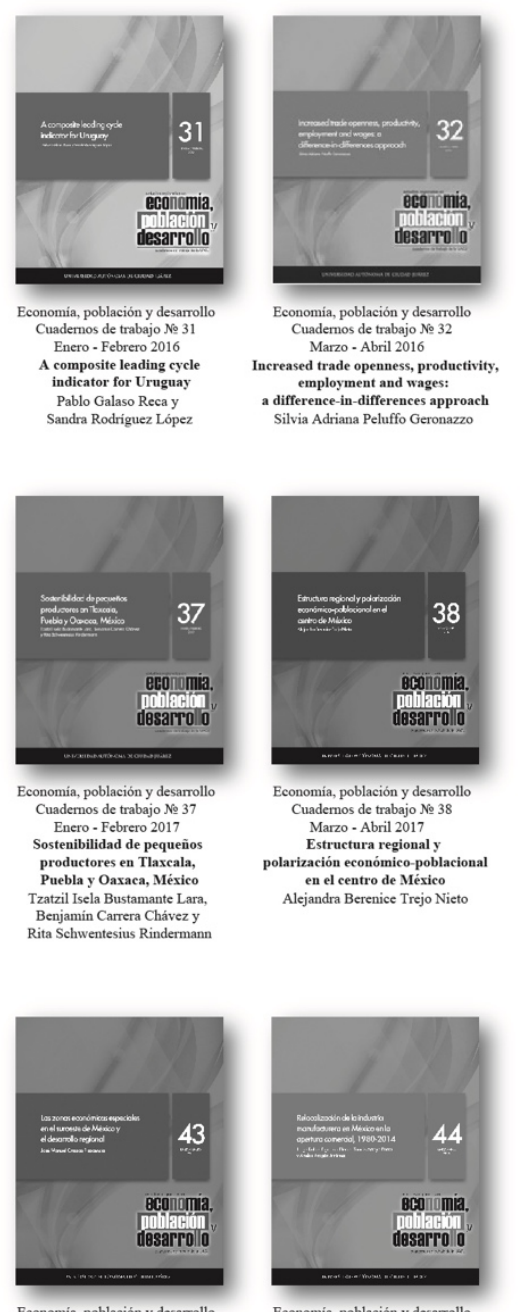

Economia, población y desarrollo Cuademos de trabajo № 43
Enero - Febrero 2018

Las zonas económicas especiale en el suroeste de México y
el desarrollo regional José Manuel Orozeo Plasser

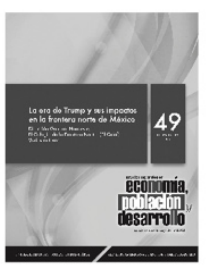

Economia, población y desarrollo
Cuademos de trabajo № 49 Enero - Febrero 2019 La era de Trump y sus impactos
en la frontera norte de México Dirección Gencral Noroes
Varios autores
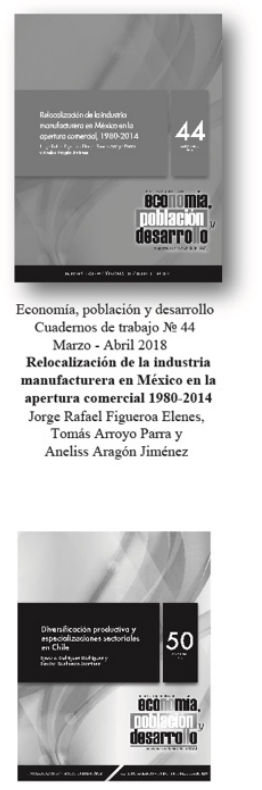

Economia, población y desarrollo Marzo - Abril 2019 Diversificación productiva y
eppecializaciones sectoriales en Chile Ignacio Rodriguez Rodriguez

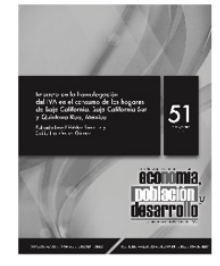
Mayo - Junio 2019

Impacto de la homologación del IV
en el consumo de los hogares de Rolando Israel Valdez Ramirez
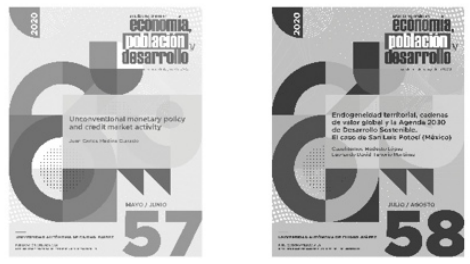

Economia, población y desarrollo
Cuademos de trabajo Ne 55 Enero - Febrero 2020 La estrategia urbanizadora de un espacio rural.
El caso de Matatlan, Mexice. Javier Renteria Vargas,
Maria Evangelina Salinas Escobar, Maria Evangelina Salinas Escobar,
Maria Teresa Renteria Rodriguez Maria Teresa Rentería Rodriguez
Ammando Chávez Hemández
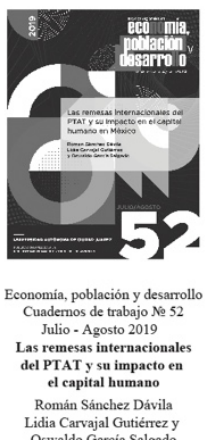

Economía, población y desarrollo
Cuadernos de trabajo № 56 Marzo - Abril 2020 Indicador integral de dotación de federativas de México, 2005-2015 Aneliss Aragón Jiménez y
Jorge Rafael Figueroa Elenes
Economia, población y desarrollo
Cuadernos de trabajo Baja Califoria, Baja Califoria Sur
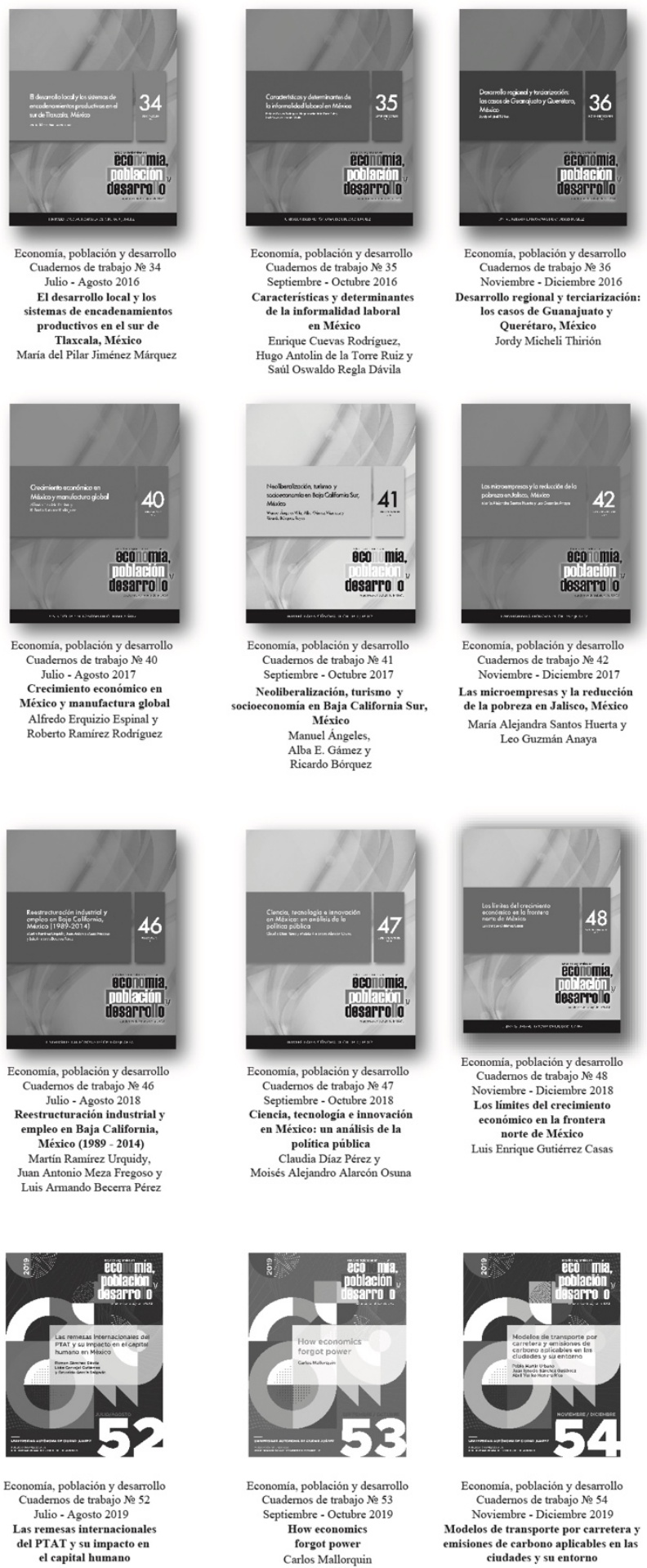

Economia, población y desarrollo Noviembre - Diciembre 2019 Modelos de transporte por carretera y
misiones de carbono aplicables en las ciudades $y$ su entorno
cution Pablo Martin Urbano, Juan Ignacio Sánchez Gutién
y Abril Yuriko Herrera Rios
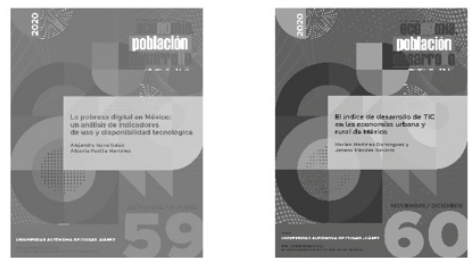

Economia, población y desarrollo
Cuademos de trabajo № 59 Septiembre - Octubre 2020 La pobreza digital en Mérico:
un anailisis de indicadores de uso an analisis de indicadores de us
disponibilidad tecnologica Alejandro Nava Galán y
Albania Padilla Martinez
Economia, población y desarrollo Noviembre - Diciembre 2020 ndice de desarrollo de TIC en las economias
urbana y rural de México Marlen Martinez Dominguez y 


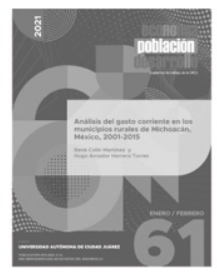

Economía, población y desarrollo
Cuademos de trabajo № 61 Enero - Febrero 2021 Análisis del gasto corriente en los municipios rurales de Michoaci
Mérico, 2001-2015 Hugo Amador Herrera Torre

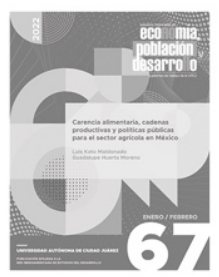

Economia, población y desarro Cuademos de trabajo №
Enero - Febrero

Carencia alimentaria, cadenas
productivas y politicas públicas par

Luis Kato Maldonado y
Guadalupe Huerta Moreno

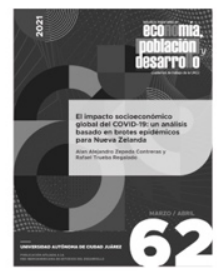

Economia, población y desarrollo Cuademos de trabajo
Marzo - Abril 2021 El impacto socioeconómico
global del COVID-19: an analisis basado
brotes epidémicos par
Nueva Zelanda Alan Alcjandro Zepeda Contreras Rafael Trueba Regalado

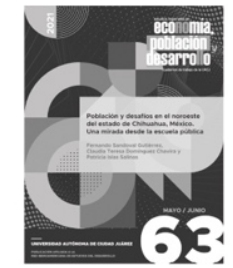

Economia, población y desarroll

Mayo - Junio 2021

Población y desafios en el noroeste
del estado de Chihuahua, México.

Una mirada desde
esceuela pública

Fermando Sandoval Gutiérrez, Claudia Teresa Dominguez Chavira
y Patricia Islas Salinas
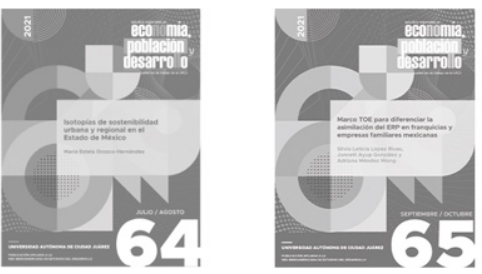

Economia, población y desarrol Cuademos de trabajo № 64 Isotopias de sostenibilida urbana y regional
en el Estado de México

Maria Estela Orozeo-Hemández
Economia, población y desarrollo Cuademos de trabajo Ne 65
Septiembre - Octubre Marco TOE para diferenciar la
similación del ERP en franquisiss Silvia Leticia López Rivas,
Jannet Ayup Gonzalezy
Adriana Mendez Wong asimilación del ERP en franquisias

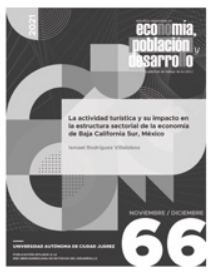

Economia, población y desarrollo Noviembre - Diciembre La actividad turistica y su impacto en la estructura sectorial de la
conomia de Baja California Sur, Méxice Ismael Rodriguez Villalobos 


\section{Para el documento general:}

Tipo de letra: Times New Roman.

Tamaño: 11 puntos.

Interlineado: 1.5 espacios.

Títulos y subtítulos:

El texto principal en 11 puntos. Títulos 12 puntos (en resaltado). Subtítulos 11 puntos. Cada título y subtítulo deberá numerarse bajo el siguiente orden: 1, 1.1, 2, 2.1, 2.2...

La extensión máxima de los cuadernos de trabajo será de 40 cuartillas.

La primera vez que se emplee una sigla en el texto se especificará primero su equivalencia completa y después la sigla.

\section{Hoja de presentación:}

Título:

14 puntos, centrado, resaltado.

Nombre de autor(es):

12 puntos

Resumen y abstract:

Debe incluir resumen en español y abstract (diez puntos), no mayor a 250 palabras

Palabras clave:

Incluir entre tres y cinco palabras clave, en español e inglés

Referencia del autor o autores:

Institución de adscripción, grado académico y líneas-grupos de investigación que desarrolla y a los que pertenece.

\section{Sistema de referencia de citas:}

Harvard-APA

Las citas bibliográficas en el texto deberán incluir entre paréntesis sólo el apellido del autor, la fecha de publicación y el número de página; por ejemplo: (Quilodrán, 2001: 33).

\section{Notación en sección de bibliografía y fuentes de información:}

Se deberá incluir al final del texto. Toda referencia deberá estar mencionada en el texto o notas de pie de página.

Cada referencia iniciará con el primer apellido o los apellidos, luego el nombre del autor, y después, entre paréntesis, el año de publicación seguido de un punto. Ejemplos:

Se deberá incluir al final del texto. Toda referencia deberá estar mencionada en el texto o notas de pie de página.

Cada referencia iniciará con el primer apellido o los apellidos, luego el nombre del autor, y después, entre paréntesis, el año de publicación seguido de un punto. Ejemplos: 
Artículo:

Ros, Jaime (2008). "La desaceleración del crecimiento económico en México desde 1982", en Trimestre Económico, vol. 75, núm. 299, pp. 537-560.

Libro:

Villarreal, René (2005). Industrialización, competitividad y desequilibrio externo en México.

Un enfoque macroindustrial y financiero (1929-2010), México, Fondo de Cultura Económica.

Capítulo de libro:

Castillo, Manuel Ángel (2003). "La política de inmigración en México: un breve recuento", en Manuel Ángel Castillo, Alfredo Lattes y Jorge Santibáñez (coords.), Migración y fronteras, Tijuana,

El Colegio de la Frontera Norte / Asociación Latinoamericana de Sociología / El Colegio de México, pp. 425-451.

\section{Notas de pie de página:}

Se utilizarán para hacer indicaciones complementarias, aclaraciones o ampliación de una explicación. La nota de pie de página en Times New Roman, 10 puntos.

\section{Tipología de imágenes dentro del texto:}

Cuadro

Gráfica

Diagrama

Mapa

Figura

Todas las imágenes deben ser numeradas y mencionadas dentro del texto. A toda imagen debe incluirse la fuente.

Las indicaciones de la imagen: tipo y número de imagen, título de imagen y fuente se escriben en 10 puntos. En el texto poner como imagen los mapas, figuras, gráficas y diagramas -con el ánimo de no perder el formato realizado por el autor.

\section{Ecuaciones y fórmulas:}

Si se utilizan ecuaciones o fórmulas deberá utilizarse el editor de ecuaciones de Word y numerarse.

\section{Envío de trabajos}

Los trabajos deben ser enviados a la dirección de correo: lgtz@uacj.mx. Con el Dr. Luis Enrique Gutiérrez Casas, editor de esta publicación.

La aceptación de cada colaboración dependerá de la evaluación de dos dictaminadores especialistas en la materia que se conservarán en el anonimato, al igual que el autor (autores) para efectos de la misma. 


\section{For General Document:}

Font type: Times New Roman.

Size: font size 11.

Paragraph: 1.5 line spacing.

Titles and subtitles: Main text font size 11. Titles font size 12 (Bold). Subtitles font size 11.

Each title and subtitle should be numbered in the following order: 1, 1.1, 2, 2.1, 2.2...

The maximum length of the workbooks will be 40 pages.

The first time an abbreviation is used in the text will be specified first complete equivalence and then stands.

\section{Front cover:}

Title:

Font size 14, centered, Bold.

Author name(s):

Font size 12.

Abstract:

It should include abstract in Spanish and abstract (font size 10), no more than 250 words.

Keywords:

Include three to five keywords, in Spanish and English.

Reference of author:

Institution of affiliation, academic degree and line-developed by research groups and belonging.

\section{Bibliographical appointment system:}

Harvard-APA

Citations in the text should include between parentheses only the author's name, publication date and page number, for example:

(Quilodrán, 2001: 33).

\section{Notation about Bibliography section and Information fonts:}

Should be included at the end of the text. All references must be mentioned in the text or footnotes page.

Each reference starts with the first name or last name, then the name of the author, and then, in parentheses, the year of publication followed by a period. Examples:

Article:

Ros, Jaime (2008). "La desaceleración del crecimiento económico en México desde 1982", en Trimestre Económico, vol. 75, núm. 299, pp. 537-560. 


\section{Editorial Guidelines}

Book:

Villarreal, René (2005). Industrialización, competitividad y desequilibrio externo en México. Un enfoque macroindustrial y financiero (1929-2010), México, Fondo de Cultura Económica.

Book chapter:

Castillo, Manuel Ángel (2003). "La política de inmigración en México: un breve recuento", en Manuel Ángel Castillo, Alfredo Lattes y Jorge Santibáñez (coords.), Migración y fronteras, Tijuana, El Colegio de la Frontera Norte / Asociación Latinoamericana de Sociología / El Colegio de México, pp. 425-451.

\section{Footnotes:}

Must be used to make additional indications, clarification or expansion of an explanation. The footnotes must be in Times New Roman, font size 10 .

\section{Image typology inside text:}

Picture

Graph

Diagram

Map

Figure

All images must be numbered and mentioned in the text, should include the source image. The indications of the image: type and number of image, image title and source are written in 10 font size. In the text set as image maps, figures, graphs and charts-with the intention of not losing the formatting by the author.

\section{Equations and Formulae:}

When using equations or formulas should be used in Microsoft Word equation editor and numbered.

\section{Paper sending}

Entries must be sent to the email address: lgtz@uacj.mx. With Dr. Luis Enrique Gutiérrez Casas, editor of this publication.

Acceptance of each collaboration will depend on the evaluation of two examiners skilled in the art to be kept anonymous, like the author(s) for the same purposes. 
Publicación afiliada a la

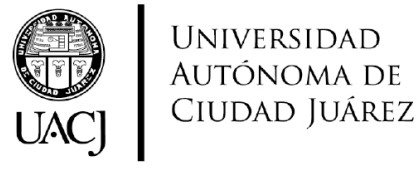

Red
RIED

Esta obra se editó y terminó de imprimir en Ciudad Juárez, Chihuahua, México 
